\title{
Multi-User Diversity in a Spectrum Sharing System
}

\author{
Tae Won Ban, Student Member, IEEE, Wan Choi, Member, IEEE, \\ Bang Chul Jung, Member, IEEE, and Dan Keun Sung, Senior Member, IEEE
}

\begin{abstract}
We investigate the effects of multi-user diversity in a spectrum sharing system where secondary users restrictively utilize a spectrum licensed to primary users only if interference perceived at primary users is regulated below a predetermined level. This interference regulation affects the characteristics of multiuser diversity gains previously known in non-spectrum sharing systems. Our numerical and analytical results show that the multiuser diversity gain in a spectrum sharing system increases differently according to conditions given by the transmit power of secondary users, $P$, and a predetermined interference temperature, $Q-$ if $P$ is sufficiently larger than $Q$, the multiuser diversity gain in terms of capacity scales like $\log _{2}\left(\mathcal{W}\left(N_{s}\right)\right)$ similarly to a previously known scaling law in the non-spectrum sharing systems, where $\mathcal{W}(\cdot)$ and $N_{s}$ denote a Lambert $W$ function and the number of secondary transmitters, respectively. However, the scaling law of multiuser diversity gain becomes $\log _{2}\left(N_{s}\right)$ as $P$ becomes sufficiently larger such that $P \gg Q N_{s}$.
\end{abstract}

Index Terms-Spectrum sharing, cognitive radio, multiuser diversity.

\section{INTRODUCTION}

A $\mathrm{N}$ exclusive assignment of frequency spectrum in radio communications causes spectral inefficiency because users rarely utilize all the assigned frequency spectrum at a certain time and a specific location as shown in the measured data [1]- [3]. This observation motivated a spectrum policy task force (SPTF) to develop a new concept of spectrum sharing to improve spectral efficiency and mitigate the shortage of the available frequency spectrum. In a spectrum sharing system, secondary users are allowed to utilize the spectrum licensed to primary users only if interference caused by the secondary users is regulated below a predetermined level. The maximum allowable interference level at the primary users' receiver is called interference temperature [1] and guarantees reliable operation of the primary users regardless of secondary users' spectrum utilization. This spectrum sharing mechanism based on the interference temperature is a more aggressive cognitive radio (CR) technique, compared to the $\mathrm{CR}$ technique

Manuscript received March 7, 2008; revised May 6, 2008; accepted June 18, 2008. The associate editor coordinating the review of this paper and approving it for publication was N. Arumugam.

This research was supported in part by the MKE (The Ministry of Knowledge Economy), Korea, under the ITRC (Information Technology Research Center) support program supervised by the IITA (Institute for Information Technology Advancement) (IITA-2008-C1090-0803-0002).

T. W. Ban and D. K. Sung are with the School of Electrical Engineering and Computer Science, KAIST, Daejeon, Korea (e-mail: \{twban@cnr, dksung@ee\}.kaist.ac.kr).

B. C. Jung is with KAIST Institute for IT Convergence, Daejeon, Korea (e-mail: bcjung@kaist.ac.kr).

W. Choi (corresponding author) is with the School of Engineering, Information and Communications University (ICU), Daejeon, Korea (e-mail: wchoi@icu.ac.kr).

Digital Object Identifier 10.1109/T-WC.2009.080326 being standardized in IEEE 802.22 [6] where secondary users use the licensed spectrum only when the primary users are idle [3]- [6].

Based on promising applications of spectrum sharing systems in future radio communications, there have been active studies on spectrum sharing systems [7]- [9]. Capacity in the absence of channel fading is analyzed in [7] and [8] when received interference power at a primary user is constrained. Fading effects on capacity of a spectrum sharing system are analyzed by using Rayleigh and Nakagami fading models when there exist multiple primary users [9]. In an analytical point of view, multiple primary receivers result in lower interference temperature compared to a single primary user case.

On the other hand, multiple secondary transmitters raise new issues related to (secondary) user scheduling and medium access control. It is well known that opportunistic selection of a user for transmission achieves a multiuser diversity gain owing to fluctuations of fading channels [10]. For nonspectrum sharing environments, there have been many studies on characterizing the multiuser diversity gains [11]- [17]. Downlink multiuser diversity in a single cell is analyzed for a large number of users in [11]- [13]. The effects of imperfect channel side information on multiuser diversity are evaluated in [14] and [15]. Choi et al. [16] and Kim et al. [17] studied the impact of interfering signals on multiuser diversity by considering multiple antennas and multiple cells. The major lessons from these previous studies are that the multiuser diversity gain in terms of capacity grows as $\log _{2}\left(\ln \left(N_{s}\right)\right)$ for Rayleigh channel fading in a single cell system without interference, where $N_{s}$ denotes the number of users in the system, and larger fluctuations of interference signals can increase the gain.

The characteristics of multiuser diversity may be different from those in spectrum sharing systems because interference regulation affects the selection of secondary users for transmission. However, there has been no work on the multiuser diversity gain in a spectrum sharing environment. Based on this motivation, we investigate the effects of multi-user diversity in a spectrum sharing system where multiple secondary users restrictively utilize a licensed spectrum.

The rest of this paper is organized as follows. In Section II, a system model is described. In Section III, we mathematically analyze the achievable capacity of a spectrum sharing system with opportunistic scheduling. The asymptotic approximation of capacity in the high power region is also derived. In Section IV, our problem formulation and numerical analysis are extended to a case where there exist multiple primary receivers. In Section V, numerical and Monte-Carlo simulation 


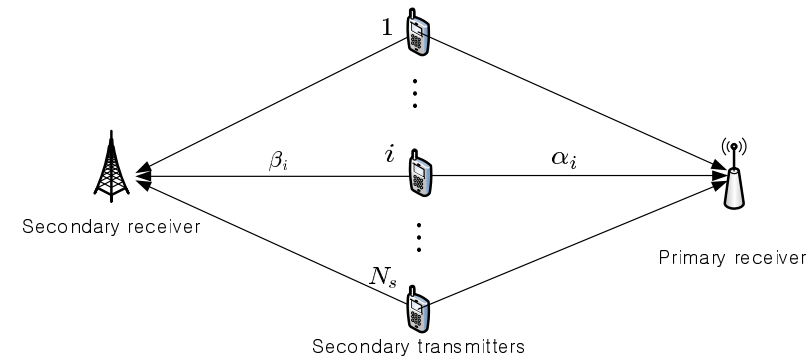

Fig. 1. System model.

results are shown and the accuracy of the numerical results is verified. Finally, conclusions are drawn in Section VI.

\section{System Model}

We consider a spectrum sharing communication system where $N_{s}$ secondary transmitters utilize a spectrum licensed to a primary user, as shown in Fig. 1. In the spectrum sharing system, any data transmission of secondary transmitters resulting in a higher interference level than an interference temperature at the primary user is not allowed. The interference temperature $Q$ represents the maximum allowable interference power level at the primary user. The channel gains from the $i$-th secondary transmitter to the primary receiver and to a target secondary receiver are denoted by $\alpha_{i}$ and $\beta_{i}$, respectively. They are assumed to be independent and identically distributed (i.i.d.) exponential random variables with unit mean in independent Rayleigh fading channels. This unit mean simplifies mathematical analysis, while other mean values capture the effect of propagation path loss [9]. It is also assumed that all the secondary transmitters have the perfect information of interference channel gains, $\alpha_{i}$. The secondary transmitters are able to obtain the information by direct feedback from the primary receiver [9] or indirect feedback from a third-party such as a band manager which mediates between the primary and secondary users [18]. As an alternative to the feedback schemes, the secondary transmitters can obtain the information through periodic sensing of pilot signal from the primary receiver assuming the channel reciprocity [19]. Despite the burden on the primary users, spectrum sharing based on the interference temperature can improve spectral efficiency more aggressively than other cognitive radio techniques [20].

In the spectrum sharing system, all the secondary transmitters which want to transmit data to the target secondary receiver sound the interference channel $\alpha_{i}$, and compute the maximum allowable transmit power $P_{i}^{t x}$ according to $\alpha_{i}$ to satisfy the interference temperature constraint at the primary user. Then, each secondary transmitter reports its transmit power $P_{i}^{t x}$ to the secondary receiver so that the secondary receiver selects the best secondary transmitter in terms of the received signal-to-noise power ratio (SNR) $\beta_{i} P_{i}^{t x} / \sigma^{2}$ where $\sigma^{2}$ is the variance of white Gaussian noise. Interference from primary users is not considered. Even though this paper ignores the detailed operation and protocol between primary transmitters and primary receivers as done in other papers [7]- [9], the interference from primary transmitters can be translated into the noise term under an assumption that interference from the primary transmitters follows a white
Gaussian distribution, which can be justified by the Central Limit Theorem (CLT) if there are many primary transmitters.

\section{Achievable Capacity of a Spectrum Sharing SySTEM With OPPORTUNISTIC USER SELECTION}

A secondary transmitter uses its maximum allowable power within its peak power constraint while satisfying the interference temperature requirement perceived at the primary user. That is, a secondary transmitter allocates its peak power for transmission if the interference temperature is satisfied with its peak power. Otherwise, it adaptively adjusts its transmit power to the allowable level so that the interference perceived at the primary receiver is maintained as a given interference temperature level $Q$. Correspondingly, the transmit power of the $i$-th secondary transmitter is given by

$$
P_{i}^{t x}= \begin{cases}P, & \alpha_{i} \leq \frac{Q}{P} \\ \frac{Q}{\alpha_{i}}, & \alpha_{i}>\frac{Q}{P}\end{cases}
$$

where $P$ represents the peak power of secondary transmitters. The adjusted power of the $i$-th secondary transmitter is used for sending data to a target secondary receiver. Thus, the received SNR at the target secondary receiver is given by

$$
\gamma_{i}=\frac{P_{i}^{t x} \beta_{i}}{\sigma^{2}}=\left\{\begin{array}{ll}
P \beta_{i}, & \alpha_{i} \leq \frac{Q}{P} \\
\frac{Q \beta_{i}}{\alpha_{i}}, & \alpha_{i}>\frac{Q}{P}
\end{array},\right.
$$

where the variance of white gaussian noise is set to be one for simplicity of analysis so that $P$ and $Q$ can also be considered as the transmit SNR and interference temperature-to-noise power ratio, respectively.

Let $Y=Q \beta_{i}$ and $Z=\alpha_{i}$ in Eq. (2). Then, the probability density function (PDF) of the received SNR at the secondary receiver from the $i$-th secondary transmitter is obtained as

$$
\begin{aligned}
f_{\gamma_{i}}(x) & =\frac{1}{P}\left(1-e^{-\frac{Q}{P}}\right) e^{-\frac{x}{P}}+\int_{\frac{Q}{P}}^{\infty} z f_{Y}(x z) f_{Z}(z) d z \\
& =\frac{1}{P}\left(1-e^{-\frac{Q}{P}}\right) e^{-\frac{x}{P}}+\frac{Q}{P} \frac{Q+P+x}{(Q+x)^{2}} e^{-\frac{Q+x}{P}}
\end{aligned}
$$

and the cumulative density function (CDF) of $\gamma_{i}$ is given as

$$
\begin{aligned}
F_{\gamma_{i}}(x) & \triangleq \int_{0}^{x} f_{\gamma_{i}}(z) d z \\
& =\left(1-e^{-\frac{Q}{P}}\right)\left(1-e^{-\frac{x}{P}}\right)+e^{-\frac{Q}{P}}-\frac{Q e^{-\frac{Q+x}{P}}}{Q+x} .
\end{aligned}
$$

Then, the secondary receiver selects a secondary transmitter with the best channel quality among $N_{s}$ secondary transmitters. The received SNR of the selected secondary transmitter $\gamma_{\max }$ is obtained as

$$
\gamma_{\max }=\max _{1 \leq i \leq N_{s}} \gamma_{i}
$$

and its PDF is given by

$$
f_{\gamma_{\max }}(x)=N_{s} f_{\gamma_{i}}(x) F_{\gamma_{i}}(x)^{N_{s}-1} .
$$

Using Eq. (6), the overall average achievable capacity is obtained by

$$
C \triangleq \mathbb{E}\left[\log _{2}\left(1+\gamma_{\max }\right)\right]=\int_{0}^{\infty} \log _{2}(1+x) f_{\gamma_{\max }}(x) d x .(7)
$$


We need to rely on a numerical evaluation of Eq. (7) because a closed form is not available. However, it is difficult to fully understand the effects of main parameters such as $P, Q$, and $N_{s}$ on the capacity by a numerical evaluation. Thus, we asymptotically analyze the capacity to understand the effects of such parameters in spectrum sharing environments on the multiuser diversity gain.

\section{A. Asymptotic Analysis in the High Power Region}

In the low power region where $P \ll Q$, it is obvious that the effect of interference temperature $Q$ disappears because the probability $\operatorname{Pr}\left[\alpha_{i}>\frac{Q}{P},\right]$ in Eq. (1) is negligible. Thus, we focus on the high power region where the effect of $Q$ is distinct.

Theorem 1: For $P \gg Q$, the average achievable capacity is approximated as

$$
C \approx C_{1}^{H}=\log _{2}\left(1+P \mathcal{W}\left(\frac{N_{s} Q}{P}\right)-Q\right)
$$

as $N_{s}$ goes to infinity.

Proof: For the high power region where $P \gg Q$, the PDF and CDF in Eqs. (3) and (4) are approximated, respectively, as

$$
\begin{aligned}
& f_{\gamma_{i}}(x) \approx f_{\gamma_{i}}^{H}(x)=\frac{Q}{P} e^{-\frac{x}{P} \frac{Q+P+x}{(Q+x)^{2}}} \\
& F_{\gamma_{i}}(x) \approx F_{\gamma_{i}}^{H}(x)=1-\frac{Q}{Q+x} e^{-\frac{x}{P}}
\end{aligned}
$$

where the approximations come from ignoring $\operatorname{Pr}\left[\alpha_{i} \leq \frac{Q}{P}\right]$ in Eq. (2), using the Taylor's series of $e^{-\frac{Q}{P}}$, and taking the smallest order of $\frac{Q}{P}$ by neglecting higher order terms. From the extreme value theory [22], [23], it is known that $\gamma_{\max }=$ $\max _{1 \leq i \leq N_{s}} \gamma_{i}$ converges to $l_{N_{s}}$ when $N_{s}$ is sufficiently large because

$$
\begin{aligned}
\lim _{x \rightarrow \infty} \frac{1-F_{\gamma_{i}}^{H}(x)}{f_{\gamma_{i}}^{H}(x)} & =\lim _{x \rightarrow \infty} \frac{1-1+\frac{Q}{Q+x} e^{-\frac{x}{P}}}{\frac{Q}{P} e^{-\frac{x}{P} \frac{Q+P+x}{(Q+x)^{2}}}} \\
& =\lim _{x \rightarrow \infty} \frac{P(Q+x)}{Q+P+x}=P>0,
\end{aligned}
$$

and $l_{N_{s}}$ is obtained as

$$
\begin{aligned}
F_{\gamma_{i}}^{H}\left(l_{N_{s}}\right) & =1-\frac{Q}{Q+l_{N_{s}}} e^{-\frac{l_{N_{s}}}{P}}=1-\frac{1}{N_{s}} \\
\rightarrow \quad l_{N_{s}} & =P \mathcal{W}\left(\frac{N_{s} Q}{P} e^{\frac{Q}{P}}\right)-Q \\
& \approx P \mathcal{W}\left(\frac{N_{s} Q}{P}\right)-Q,
\end{aligned}
$$

where $\mathcal{W}(\cdot)$ denotes a Lambert $\mathrm{W}$ function [24] and the approximation in Eq. (11) comes from the fact that $P \gg Q$. Therefore, as $N_{s}$ goes to infinity, the capacity in Eq. (7) is approximated as Eq. (8).

Theorem 1 indicates that the multiuser diversity gain in terms of capacity in a spectrum sharing environment grows as $\log _{2}\left(\mathcal{W}\left(N_{s}\right)\right)$ in the high power region.
Theorem 2: For $P \gg N_{s} Q$, the average achievable capacity is approximated as

$$
C \approx C_{2}^{H}=\log _{2}\left(1+\left(N_{s}-1\right) Q\right)
$$

as $N_{s}$ goes to infinity.

Proof: As the transmit power $P$ becomes much higher, i.e., $P \gg N_{s} Q$, the Lambert $\mathrm{W}$ function is approximated as $\mathcal{W}\left(\frac{N_{s} Q}{P}\right) \approx \frac{N_{s} Q}{P}$ because $\mathcal{W}(x) \approx x$ for $x \ll 1$. Correspondingly, the capacity $C_{1}^{H}$ in Eq. (8) can be reapproximated as

$$
\begin{aligned}
C_{1}^{H} \approx C_{2}^{H} & =\log _{2}\left(1+P\left(\frac{N_{s} Q}{P}\right)-Q\right) \\
& =\log _{2}\left(1+\left(N_{s}-1\right) Q\right) .
\end{aligned}
$$

Theorem 2 shows an interesting result that the capacity in multi-user spectrum sharing environments grows like $\log _{2}\left(N_{s} Q\right)$ as the transmit power becomes much larger such that $P \gg N_{s} Q$. It becomes more difficult to satisfy the interference regulation for a primary user when the transmit power of secondary transmitters is very high. Correspondingly, selection of a secondary transmitter is much more affected by the interference channels from secondary transmitters to the primary user rather than data channels from secondary transmitters to the target secondary receiver. Thus, the selection of a secondary transmitter is focused on selection of a secondary transmitter with the lowest interference channel in this very high power region. By selecting a secondary user with the lowest interference channel, a chance to use higher power for transmission to the target secondary receiver increases. Specifically, let $\alpha_{\min }=\min _{1 \leq i \leq N_{s}} \alpha_{i}$ denote the lowest interference channel. Then, $\alpha_{\min }$ is exponentially distributed with mean $\frac{1}{N_{s}}$, as shown in Appendix, which allows an increase in the transmit power by $N_{s}$ in average and yields a capacity increase at a rate of $\log _{2}\left(N_{s}\right)$. As a result, the scaling law of capacity changes, as shown in Theorem 2 .

\section{Extension to Multiple Primary Receivers}

In this section, we extend our results to a spectrum sharing environment with multiple primary users. Thus, there are $N_{p}$ primary users and $N_{s}$ secondary users and hence each secondary transmitter has $N_{p}$ interference channels denoted by $\alpha_{i, j}, i \in\left\{1, \cdots, N_{s}\right\}, j \in\left\{1, \cdots, N_{p}\right\}$. Then, the transmit power of the $i$-th secondary transmitter is determined by

$$
P_{i}^{t x}=\left\{\begin{array}{ll}
P, & \tilde{\alpha}_{i} \leq \frac{1}{P} \\
\frac{1}{\tilde{\alpha}_{i}}, & \tilde{\alpha}_{i}>\frac{1}{P}
\end{array},\right.
$$

where $Q_{j}$ represents a given interference temperature for the $j$-th primary receiver and $\tilde{\alpha}_{i}$ is defined as $\tilde{\alpha}_{i} \triangleq$ $\max _{1 \leq j \leq N_{p}}\left(\frac{\alpha_{i, j}}{Q_{j}}\right)$, of which CDF and PDF are obtained by

$$
F_{\tilde{\alpha}_{i}}(x)=\prod_{j=1}^{N_{p}}\left(1-e^{-Q_{j} x}\right), \quad f_{\tilde{\alpha}_{i}}(x)=\frac{d}{d x} F_{\tilde{\alpha}_{i}}(x) .
$$

Thus, the received SNR at the target secondary receiver from the $i$-th secondary transmitter is given by

$$
\gamma_{i}=\frac{P_{i}^{t x} \beta_{i}}{\sigma^{2}}=\left\{\begin{array}{cc}
P \beta_{i}, & \tilde{\alpha}_{i} \leq \frac{1}{P} \\
\frac{\beta_{i}}{\tilde{\alpha}_{i}}, & \tilde{\alpha}_{i}>\frac{1}{P}
\end{array}\right.
$$




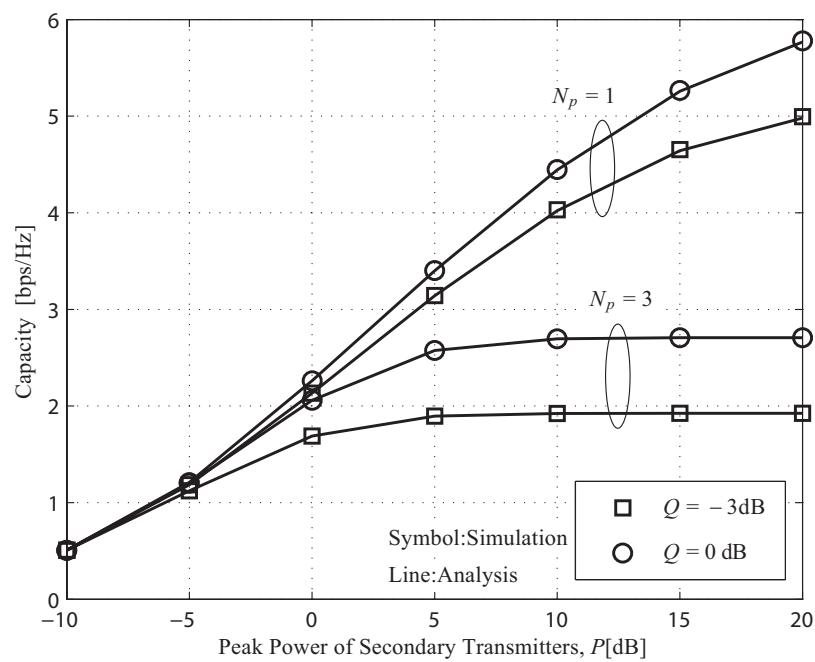

Fig. 2. Achievable capacity versus $P$ for two different $Q$ and $N_{p}$ values. $N_{s}=40$.

Let $Y=\beta_{i}$ and $Z=\tilde{\alpha}_{i}$ in Eq. (15). Then, the PDF of the received SNR from the $i$-th secondary transmitter is derived as

$$
\begin{aligned}
f_{\gamma_{i}}(x) & =F_{Z}\left(\frac{1}{P}\right) \frac{1}{P} e^{-\frac{x}{P}}+\int_{\frac{1}{P}}^{\infty} z f_{Y}(x z) f_{Z}(z) d z \\
& =\prod_{j=1}^{N_{p}}\left(1-e^{-Q_{j} / P}\right) \frac{1}{P} e^{-\frac{x}{P}}+\int_{\frac{1}{P}}^{\infty} z e^{-x z} f_{Z}(z) d z .
\end{aligned}
$$

For the identical $Q_{j}=Q, \forall j$, Eq. (16) is reduced to

$$
\begin{aligned}
f_{\gamma_{i}}(x)=\frac{1}{P} & \left(1-e^{-\frac{Q}{P}}\right)^{N_{p}} e^{-\frac{x}{P}} \\
& +\int_{\frac{1}{P}}^{\infty} z e^{-x z} N_{p} Q e^{-Q z}\left(1-e^{-Q z}\right)^{N_{p}-1} d z \\
= & \frac{1}{P}\left(1-e^{-\frac{Q}{P}}\right)^{N_{p}} e^{-\frac{x}{P}}+\frac{N_{p} Q}{P} e^{-\frac{x}{P}} \\
& \times \sum_{k=1}^{N_{p}}\left(\begin{array}{c}
N_{p}-1 \\
k-1
\end{array}\right)(-1)^{k-1} e^{-\frac{k Q}{P}} \frac{k Q+P+x}{(k Q+x)^{2}},
\end{aligned}
$$

where the second equality comes from a binomial expansion of $\left(1-e^{-Q z}\right)^{N_{p}-1}$ [21]. In addition, the CDF of $\gamma_{i}$ is given by

$$
\begin{aligned}
F_{\gamma_{i}}(x) \triangleq & \int_{0}^{x} f_{\gamma_{i}}(z) d z \\
= & \left(1-e^{-\frac{Q}{P}}\right)^{N_{p}}\left(1-e^{-\frac{x}{P}}\right)+N_{p} \sum_{k=1}^{N_{p}}\left(\begin{array}{c}
N_{p}-1 \\
k-1
\end{array}\right) \\
& \quad \times(-1)^{k-1} e^{-\frac{k Q}{P}}\left[\frac{1}{k}-\frac{Q}{Q k+x} e^{-\frac{x}{P}}\right] . \quad(18)
\end{aligned}
$$

Substituting Eqs. (17) and (18) into Eq. (6) and using Eq. (7) yield the achievable capacity.

\section{NUMERICAL RESUlts}

Fig. 2 shows the achievable capacity versus the peak power of a secondary transmitter, $P$, for two different values of interference temperature, $Q$ and the number of primary receivers,

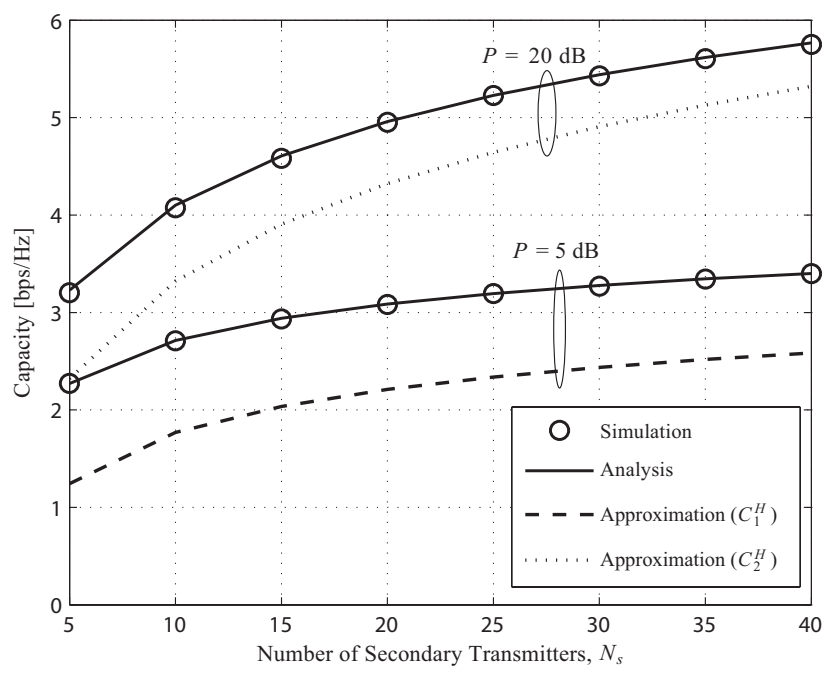

Fig. 3. Achievable capacity versus $N_{s} . P=5$ or $20 \mathrm{~dB}, Q=0 \mathrm{~dB}$, and $N_{p}=1$.

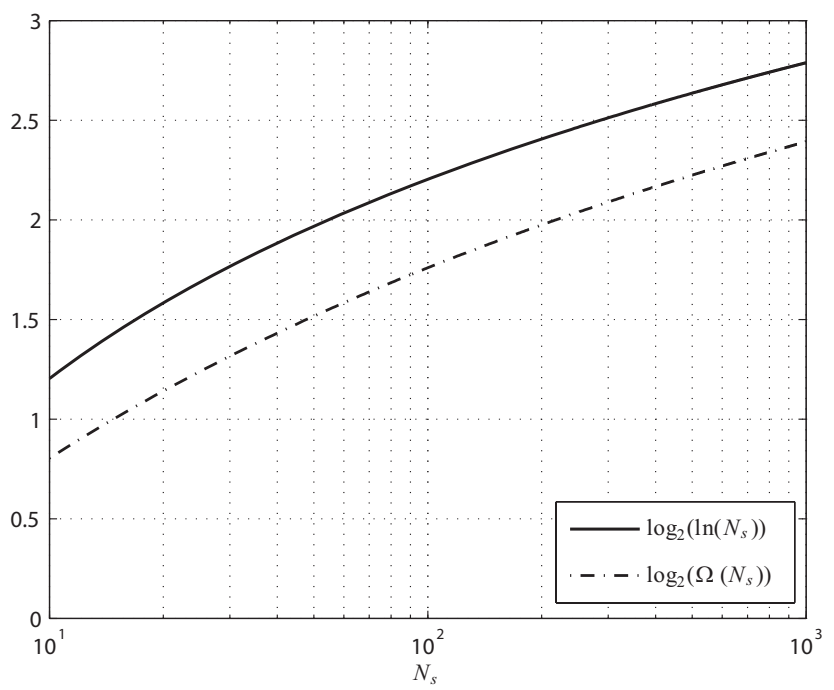

Fig. 4. The scaling of log and Lambert $\mathrm{W}$ functions.

$N_{p}$ when $N_{s}=40$. It is shown that the capacity of a multiuser spectrum sharing system grows as the transmit power of a secondary user increases, but it is saturated at a certain level unlike non-spectrum sharing systems with opportunistic user selection. Lower interference temperature results in lower achievable capacity as expected. The result also shows that capacity decreases with the number of primary receivers, $N_{p}$ since more constraints reduce the available degree of freedom at the secondary transmitters.

Fig. 3 shows the achievable capacity versus $N_{s}$ for two different values of $P$ when $Q=0 \mathrm{~dB}$ and $N_{p}=1$. It is verified that the asymptotic approximation exactly characterizes the scaling law of the multiuser diversity gain in a multi-user spectrum sharing environment even though the asymptotic capacity approximations do not coincide with the exact capacity curve. It is also verified that a scaling law of capacity is $\log _{2}\left(\mathcal{W}\left(N_{s}\right)\right)$ when $P$ is sufficiently larger than $Q$. The scaling of $\log _{2}\left(\mathcal{W}\left(N_{s}\right)\right)$ is similar to $\log _{2}\left(\ln \left(N_{s}\right)\right)$, as shown in Fig. 4. Fig. 3 also shows that the capacity grows very fast 


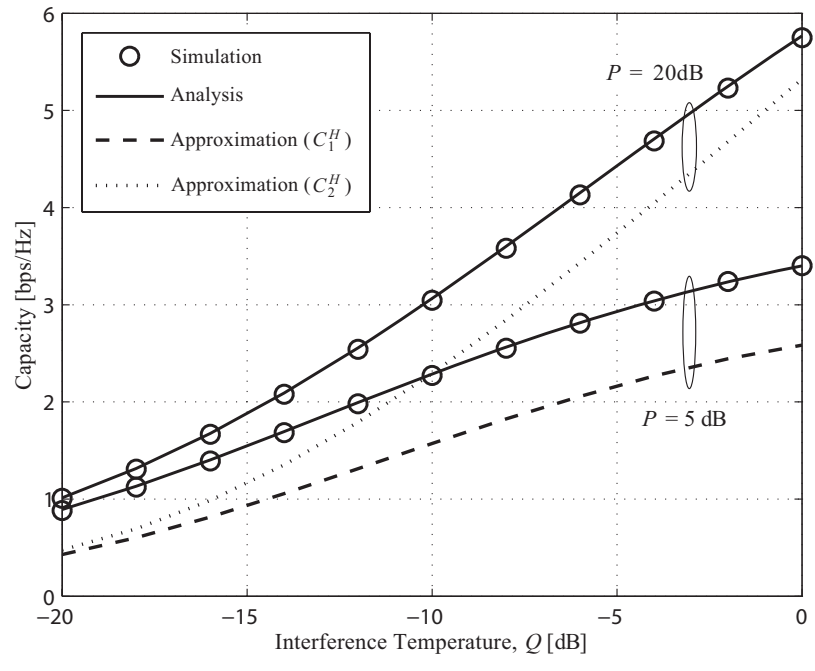

Fig. 5. Achievable capacity versus $Q . P=5$ or $20 \mathrm{~dB}, N_{s}=40$ and $N_{p}=1$.

like $\log _{2}\left(N_{s}\right)$ as $P$ becomes much larger such that $P \gg Q N_{s}$.

Fig. 5 shows the achievable capacity versus $Q$ for two different values of $P$ when $N_{s}=40$ and $N_{p}=1$. The capacity increases as $Q$ grows because the transmit power of the selected secondary transmitter increases with $Q$ as in Eq. (2). It should be noted that when $P$ is sufficiently larger than $Q N_{s}$, the capacity quickly grows as $\log _{2}(Q)$, as shown in this figure.

\section{CONCLUSION}

We analyzed the multiuser diversity gain in a spectrum sharing environment where the transmission of secondary users is regulated by a given interference temperature at a primary user. Through asymptotic analysis in the high power region, we found that the multiuser diversity gain in terms of capacity grows like $\log _{2}\left(\mathcal{W}\left(N_{s}\right)\right)$ when $P$ is sufficiently larger than $Q$. The scaling law becomes steep like $\log _{2}\left(N_{s}\right)$ as $P$ becomes much larger such that $P \gg N_{s} Q$, while the capacity scales like $\log _{2}\left(\ln \left(N_{s}\right)\right)$ in non-spectrum sharing systems with opportunistic user selection. Our results indicate that interference regulation for primary users changes the characteristics of multiuser diversity previously known in nonspectrum sharing environments. These effects are dominant in the very high power region because selection of a secondary user is much more influenced by the interference channels from secondary users to the primary user.

\section{APPENDIX}

Let $X_{\min }=\min _{1 \leq i \leq N} X_{i}$, where $X_{i}$ 's are i.i.d and exponentially distributed random variables with mean $\lambda$. Then, the cdf and pdf of $X_{\min }$ can be obtained as

$$
\begin{aligned}
F_{X_{\text {min }}}(x) & =\operatorname{Pr}\left[X_{\min } \leq x\right]=1-\prod_{1 \leq i \leq N} \operatorname{Pr}\left[X_{i}>x\right] \\
& =1-\prod_{1 \leq i \leq N} e^{-\frac{x}{\lambda}}=1-e^{-\frac{x}{\lambda / N}} \\
f_{X_{\text {min }}}(x) & \triangleq \frac{d}{d x} F_{X_{\text {min }}}(x)=\frac{N}{\lambda} e^{-\frac{x}{\lambda / N}}
\end{aligned}
$$

Therefore, it can be concluded that $X_{\min }$ is also exponentially distributed with mean $\frac{\lambda}{N}$.

\section{REFERENCES}

[1] Federal Communications Commission, "Spectrum policy task force report," ET Docket No. 02-135, Nov. 2002.

[2] D. Cabric, I. D. O'Donnell, M. S.-W. Chen, and R. W. Brodersen, "Spectrum sharing radios," IEEE Circuits and Systems Mag., vol. 6, no. 2, pp. 30-45, 2006.

[3] B. Fette, Cognitive Radio Technology. Elsevier, 2006.

[4] J. Mitola and G. Q. Maguire, "Cognitive radios: making software radios more personal," IEEE Personal Commun., vol. 6, no. 4, pp. 1318, Aug. 1999.

[5] S. Haykin, "Cognitive radio: brain-empowered wireless communications," IEEE J. Select. Areas Commun., vol. 23, no. 2, pp. 201-220, Feb. 2005.

[6] Website of IEEE 802.22 Working Group, http://www.ieee802.org/22/.

[7] M. Gastpar, "On capacity under received-signal constraints," in Proc. 42nd Annual Allerton Conf. Comm., Control Comp., Sept. 2004.

[8] M. Gastpar, "On capacity under receiver and spatial spectrum-sharing constraints," IEEE Trans. Inform. Theory, vol. 53, no. 2, pp. 471-487, Feb. 2007.

[9] A. Ghasemi and E. S. Sousa, "Fundamental limits of spectrum-sharing in fading environments," IEEE Trans. Wireless Commun., vol. 6, no. 2, pp. 649-658, Feb. 2007.

[10] R. Knopp and P. A. Humblet, "Information capacity and power control in single-cell multiuser communications," in Proc. IEEE ICC'95, June 1995.

[11] Zhouhua, Yangdacheng, Qiweishi, and MaMin, "On performance of mutiuser diversity in SISO and MIMO wireless communication," in Proc. IEEE PIMRC'03, Sept. 2003.

[12] C.-J. Chen and L.-C. Wang, "A unified capacity analysis for wireless systems with joint multiuser scheduling and antenna diversity in Nakagami fading channels," IEEE Trans. Commun., vol. 54, no. 3, pp. 469-478, Mar. 2006.

[13] G. Song and Y. Li, "Asymptotic throughput analysis for channel-aware scheduling," IEEE Trans. Commun., vol. 54, no. 10, pp. 1827-1834, Oct. 2006.

[14] Q. Ma and C. Tepedelenlioglu, "Practical multiuser diversity with outdated channel feedback," IEEE Trans. Veh. Technol., vol. 54, no. 4, pp. 1334-1345, July 2005.

[15] V. K. N. Lau and M. Jiang, "Performance analysis of multiuser downlink space-time scheduling for TDD systems with imperfect CSIT," IEEE Trans. Veh. Technol., vol. 55, no. 1, pp. 296-305, Jan. 2006.

[16] W. Choi, N. Himayat, S. Talwar, J. Y. Kim, A. Koo, J. Choi, Y. Noh, and J. Kim, "Interactions between multiuser diversity and spatial diversity techniques in an interference-limited environment," in Proc. IEEE WCNC'07, Mar. 2007.

[17] S. H. Kim, B. C. Jung, and D. K. Sung, "Effect of other-cell interference on multiuser diversity in cellular networks," in Proc. IEEE ISCIT'07, Sept. 2007.

[18] J. M. Peha, "Approaches to spectrum sharing," IEEE Commun. Mag., vol. 43, no. 2, pp. 10-12, Feb. 2005.

[19] Q. Zhao, S. Geirhofer, L. Tong, and B. M. Sadler, "Opportunistic spectrum access via periodic channel sensing," IEEE Trans. Signal Processing, vol. 56, no. 2, pp. 785-796, Feb. 2008.

[20] Website of FCC, http://www.fcc.gov/sptf/reports.html.

[21] A. Papoulis and S U. Pillai, Probability, Random Variables and Stochastic Processes. McGraw-Hill, fourth ed., 2002.

[22] H. David and H. Nagaraja, Order Statistics. Wiley, 2003.

[23] P. Viswanath, D. N. C. Tse, and R. Laroia, "Opportunistic beamforming using dumb antennas," IEEE Trans. Inform. Theory, vol. 48, no. 6, pp. 1277-1294, June 2002.

[24] I. S. Gradshteyn and I. M. Ryzhik, Table of Integrals, Series, and Products, 6th ed. San Diego, CA: Academic, 2000. 